Available online at GSC Online Press Directory

GSC Biological and Pharmaceutical Sciences

e-ISSN: 2581-3250, CODEN (USA): GBPSC2

Journal homepage: https://www.gsconlinepress.com/journals/gscbps

(CASE REPORT ARTICLE)

\title{
A rare case of metastasis from primitive breast cancer in endometrioid adenocarcinoma
}

\author{
Erra Stefania ${ }^{1,}{ }^{*}$, Frigeri Alessia ${ }^{1}$, De Luca Michele ${ }^{1}$, Zambello Luca ${ }^{1}$, Caminiti Valentina ${ }^{1}$ and Buosi \\ Roberta ${ }^{2}$
}

${ }_{1}^{1}$ Surgical Pathology Department, Santo Spirito Hospital, Casale Monferrato, Viale Giolitti 2, Italy

${ }^{2}$ Oncology Unit, Santo Spirito Hospital, Casale Monferrato, Viale Giolitti 2, Italy.

Publication history: Received on 16 June 2020; revised on 25 June 2020; accepted on 27 June 2020

Article DOI: https://doi.org/10.30574/gscbps.2020.11.3.0190

\begin{abstract}
Breast cancer is the most common cancer in women, with a high mortality rate. Genital metastasis of breast carcinoma are rare. Even if it is known that breast cancer can metastasize to many organ sites, metastasis to the uterus is uncommon and usually occurs during widespread metastatic disease, with only a limited number of such cases reported in the literature to date. Uterine metastases account for approximately $4 \%$ of genital tract metastases, with $47 \%$ of case involving as the primary site. We herein report the case of an 83-year-old female patient, who was diagnosed with endometrioid adenocarcinoma with metastases of infiltrating ductal carcinoma. Breast cancer is the most frequently diagnosed cancer globally and it is the leading cause of cancer-related death in women. Breast cancer screening programs and improved treatment of primary tumors have contributed significantly reducing overall mortality rates. Metastatic lesion are clearly secondary metastases of breast cancer in patients with disseminated disease, while other tumors can be confused with primary neoplasm. This group represents the tumors with high aggressiveness as these are less differentiated carcinomas, with a greater tendency to metastasize through the lymphatic and vascular structures.
\end{abstract}

Keywords: Primitive breast cancer; Endometrial adenocarcinoma; Infiltrating ductal carcinoma

\section{Introduction}

Breast cancer is the most common cancer in woman. Metastasis to the female genital tract from extra-genital cancers are rare.[1][2]

Breast cancer includes a number of histological subtypes the most common being invasive ductal carcinoma (IDC) and invasive lobular carcinoma (ILC).[3]

ILC is the most frequent histological type that metastasizes to the female genital tract in more than $80 \%$ of all cases. $[4][5]$.

The most frequent site of breast cancer metastasis is bone, followed by lung, pleura, soft tissue, and liver. Metastasis from breast carcinoma to the gallbladder has been reported in about 4-7\% cases. [6] The endometrium accounts for $4,7 \%$ of metastatic sites to the female genital tract from extra-genital tumors. [7]

\footnotetext{
* Corresponding author: Erra Stefania
} 
There are few reports of breast cancer cases with uterine metastasis. Among them, myometrium is more frequently involved than endometrium. The majority of breast cancer metastasis to endometrium are lobular carcinoma, with only 5 reported cases of the ductal carcinoma histotype since 1984.[8]

Genital tract metastasis in patients with known breast carcinoma can present with abnormal vaginal bleeding, but they often are asymptomatic, therefore to obtain an early diagnosis it's necessary to carry out rigorous gynecological surveillance.[9]

A central role is given to immunohistochemistry, which througth is investigation can carry out a differential diagnosis, allowing the clinician to establish an appropriate treatment.

Here we reported a rare case of invasive ductal carcinoma causing endometrium metastasis.

\section{Case report}

A 83 years old woman was complaining of postmenopausal uterine bleeding. Her medical history revealed that she was diagnosed a breast node 3 years ago.

Following a menorrhagia, a trans-vaginal ultrasound exam was performed which found an endometrial hyperplasia of $12.4 \mathrm{~mm}$.

Hysteroscopy found the uterine cavity totally occupied by a whitish, friable and bleeding vegetative formation.

A surgical removal was planned for resection of the lesion. The surgical specimen was sent in the surgical pathology laboratory for histological diagnosis. Macroscopically, the lesion was represented by an endometrial uterine mass of $\mathrm{cm}$ $7 \times 4 \times 3$.

After the microscopic examination, a moderately differentiated endometrioid adenocarcinoma was found, infiltrating the myometrium for less than half its thickness (figure 1, 2a). In the same sections, in correspondence with the perimeter of the uterine body, it was detected a poorly differentiated neoplasia whose immunophenotype (GATA3 +, cadherin E + ) is consistent with breast primitiveness (figure $2 \mathrm{~b}, 3 \mathrm{a}, 3 \mathrm{~b}$ ).

The mammographic examination previously performed detected a mass with irregular contours of $5 \mathrm{~cm}$ in which ultrasound guided core-biopsy was made. The histological diagnosis revealed an infiltrating ductal carcinoma with ER 95\%, PgR 60\%, Ki 30\%, Her-2 neu not amplified.

The case was discussed in a multidisciplinary team. Only hormone therapy with letrozolo was started considering the co-morbidity, the PS and the advanced age of the patient. This indication is in according to international Guidelines about the old patients.[10]

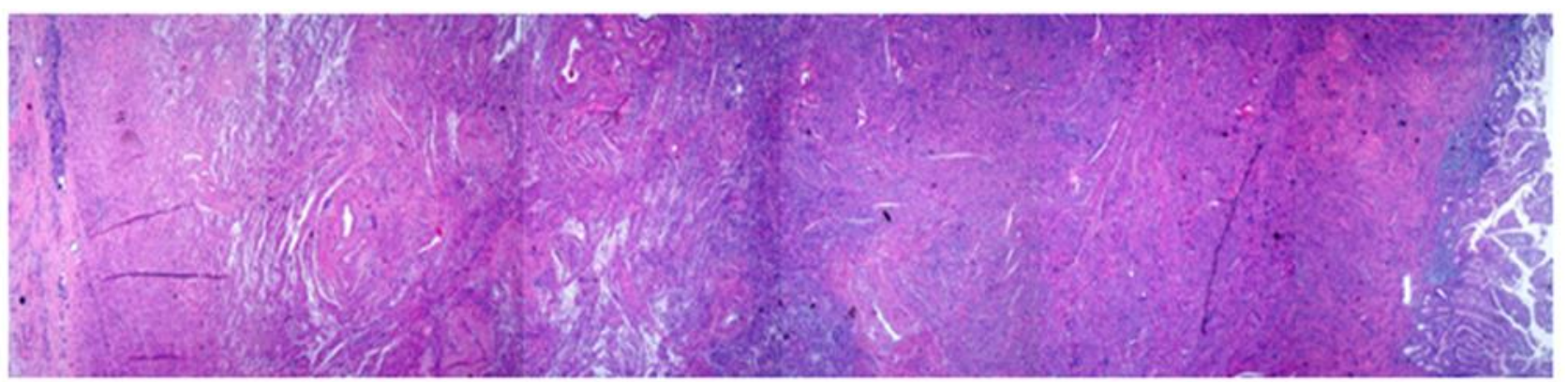

Figure 1 Hematoxylin and eosin staining of primitive breast cancer metastasis compatible with ductal histotype in endometrioid adenocarcinoma. (Magnification 4X). 


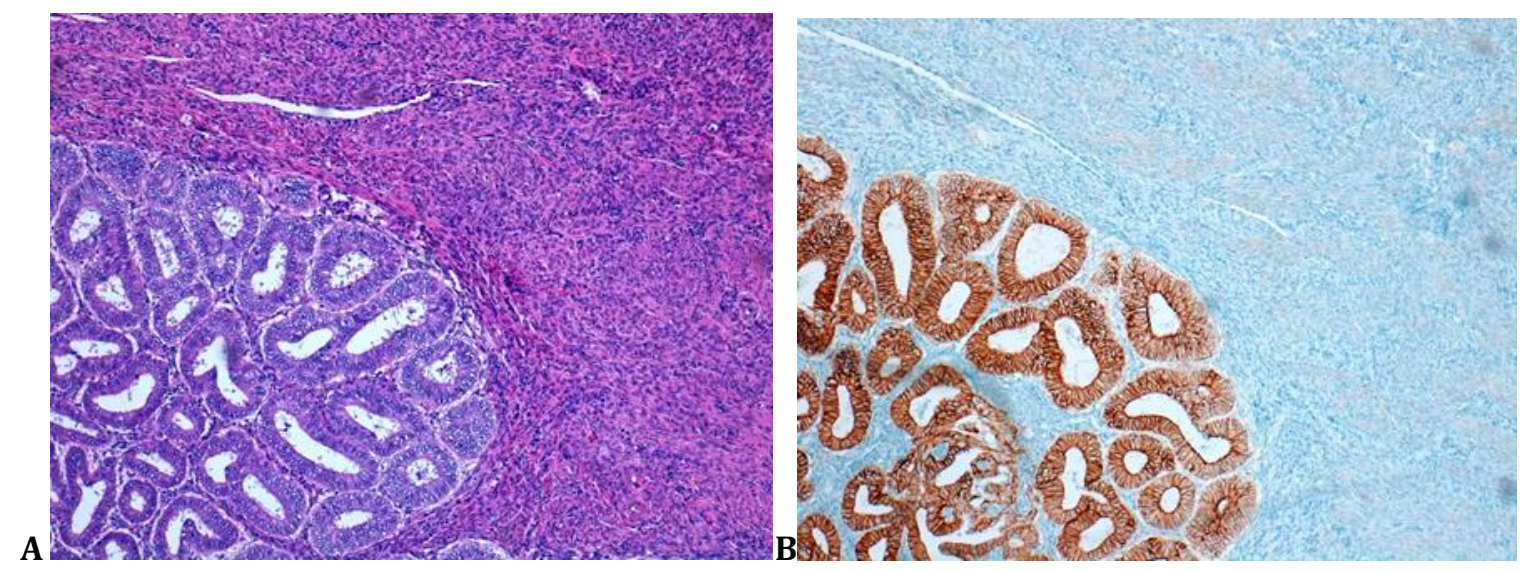

Figure 2A-2BHematoxylin and eosin staining of endometrioid adenocarcinoma infiltrating the myometrium for less than half its thickness (a); E-cadherin staining shows diffuse strong membranous pattern (b) (Magnification 10X).
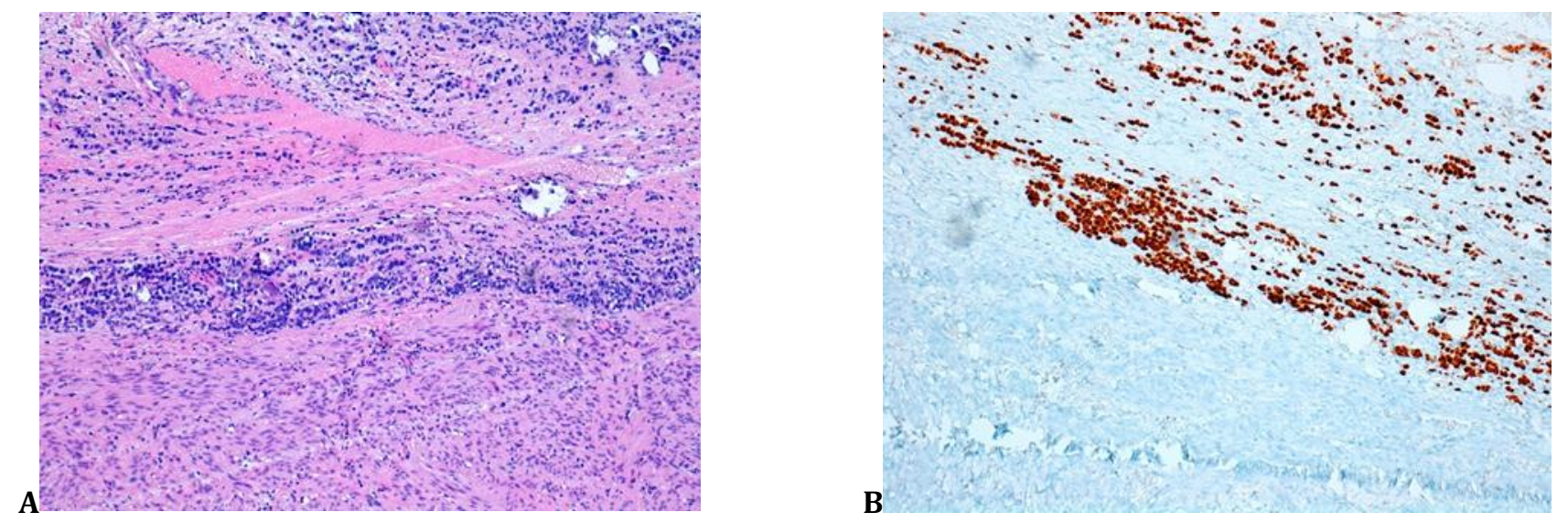

Figure 3A-3B Hematoxylin and eosin staining of ductal histotype of primitive breast cancer, poorly differentiated, infiltrating the endometrioid adenocarcinoma (a); Representative results of immunohistochemical nuclear staining of GATA3, highlighting primitive breast neoplasia (b) (Magnification 10X).

\section{Discussion}

Ductal carcinoma is the most common invasive breast cancer type. It represents between $70 \%$ and $80 \%$ of all breast malignant neoplasia. In general the initial forms of breast cancer are asymptomatic, any palpable or visible nodules are to be sought; half of the cases of breast cancer occur in the upper external quadrant of the breast. The diagnosis is carried out by mammography, with a recommended biopsy or fine needle aspiration cytology for morphological diagnosis.

Fever than $10 \%$ of patients have metastatic disease at time of presentation. Metastasis to the uterus is a rare event, with only a limited number of such cases in the literature to date. [8,9]

Mazur et al. analized 52 cases of breast cancer metastasis to gynecologic organs and the result indicated that the ovaries were affected in $86.5 \%$ of cases (45/52), endometrium in $3.8 \%(2 / 52)$, vagina in $5.8 \%(3 / 52)$, and the vulva and cervix in $1.95 \% .(1 / 52)[12]$

In addiction lobular carcinoma more frequently spreads to gynecologic organs than ductal one.

Metastasis of breast ductal infiltrating cancer to endometrium are rarely been reported to literature. We found five cases including the one presented here, among these five cases, two were TNBC (triple negative breast cancer), three, including ours, were ER or PR positive. [13] 
Breast cancer represents one of the leading causes of mortality and morbidity among females. It is regulated in part by different classes of adhesions molecules, one of which is known as cadherins.

Cadherins have been found to regulate breast cancer in positive as well as negative fashions,whereby E-Cadherin (CDH1) and N-Cadherin(CDH2) contribute significantly to wards transitioning from epithelial stale to mesenchymal state (EMT) and enacting the abnormal cells to invade and metastasize nearby and distant tissue.[14]

E-Cadherin promotes metastasis in different modes of invasive ductal carcinomas; while loss of E-Cadherin increases invasion and also reduces cancer cell proliferation and survival, circulating tumor cell number, seeding of cancer cells in distant organs and metastasis outgrowth. [15]

In these cases E-cadherin positivity allows to evaluate the breast primitiveness of secondary neoplastic localizations.

Metastatic involvement of the endometrium should be considered when vaginal bleeding or an enlarged uterus are detected. Pathological microscopic diagnosis is indispensable to distinguish metastasis from primary lesion, because the oncologic treatment differs.

Systemic chemotherapy and hormonal treatment can play a central role in these patients by improving survival and quality of life.

\section{Conclusion}

Metastatic breast cancer in the uterus often poses diagnostic problems for both the clinician and the pathologist. It's imperative to differentiate breast cancer metastasis from primary carcinoma of the genital tract; these differentiation may result difficult because there aren't specific symptoms at presentations, long disease-free interval, TC or MRI scan are not enough for differential diagnosis. For the later, only histological and immunohistochemical analysis are really useful for a reliable differential diagnosis.[16].

\section{Compliance with ethical standards}

\section{Disclosure of conflict of interest}

Authors declare that there are no conflicts of interest in connection with this paper, and the material described is not under publication or consideration for publication elsewhere.

\section{Statement of informed consent}

Informed consent has been obtained from the subject included in the study.

\section{References}

[1] Kumar NB and Hart WR.(1984). Metastases to the uterine corpus from extragenital cancers: a clinicopathologic study of 63 cases. Cancer, 50(10), 2163-2169.

[2] Mazur MT, Hsueh S and Gersell DJ. (1984).Metastases to the female genital tract: analysis of 325 cases. Cancer, 53(9), 1978-1984.

[3] Briki R, Cherif O, Bannour B, Hidar S, Boughizane S and Khairi H. (2018). Uncommon metastases of invasive lobular breast cancer to the endometrium: a report of two cases of the literature. Pan.African Medical Journal, 30, 268.

[4] Harris M, Howell A and Chrissohou M.(1984). A comparison of the metastatic pattern of infiltrating lobular carcinoma and infiltrating duct carcinoma of the breast. British journal of cancer, 50(1), 23-30.

[5] Ustaalioglu BBO, Bilici A, Seker M et al. (2009). Metastasis of lobular breast carcinoma to the uterus in a patient under anastrozole therapy. Onkologie, 32(7), 424-426.

[6] Lee YT. (1983). Breast carcinoma: pattern of metastases at autopsy. J.Surg. Oncol, 23(3), 175-180.

[7] Abrams HL, Spiro R and Goldstein N. (1950). Metastases in carcinoma: analysis of 1000 autopsied cases. Cancer, 3 (1), 74-85. 
[8] Rahmani M, Nili F and Tabibian E. (2018). Endometrial metastasis from ductal breast carcinoma. A case report with literature review. American Journal of Case Reports, 19, 494-499.

[9] Bogliolo S, Morotti M, Valenzano Menada M, Fulcheri E, Musizzano Y and Casabona F. (2010). Breast cancer with syncronous massive metastasis in the uterine cervix: a case report and review of the literature. Arch Gynecol Obstet, 281 (4), 769-733.

[10] William J. Gradishar MD, Benjamin O. Anderson MD, Jame Abraham MD et Al. (2020). Breast Cancer, version 3.2020, NCCN Clinical Practice Guide Lines in Oncology,118(4).

[11] Roses DF. (2005). Breast cancer 2nd edition, Elsevier, Pennsylvania, 812.

[12] Huo Z, Gao Y, Zuo W, Zheng G and Kong R. (2015). Metastases of basal-like breast invasive ductal carcinoma to the endometrium: a case report and review of the literature. Thoracic Cancer, 6, 548-552.

[13] Lamovec J and Bracko M. (1991). Metastatic pattern of infiltrating lobular carcinoma of the breast: an autopsy study. J. Surg. Oncol,48, 28-33.

[14] Ashaie MA and Chowdhury EH. (2016). Cadherins: the superfamily critically involved in breast cancer. Curr .Pharm. Des, 22(5), 616-638.

[15] Padmanaban V, Krol I, Suahil Y, Szczerba BM, Aceto N, Bader JS and Ewald AJ. (2019). E-cadherin is required for metastasis in multiple models of breast cancer. Nature, 573 (7774), 439-444.

[16] Thouvenot A, Bizet Y, Baccar LS and Lamuraglia M.(2018). Primary breast cancer relapse as metastasis to the cervix uteri: a case report. Molecular and Clinical Oncology, 9, 96-97.

\section{How to cite this article}

Erra S, Frigeri A, De Luca M, Zambello L, Caminiti V and Buosi R. (2020). A rare case of metastasis from primitive breast cancer in endometrioid adenocarcinoma. GSC Biological and Pharmaceutical Sciences, 11(3), 261-265. 